\title{
Avoiding crisis in communication: a computer-supported training approach for emergency management
}

\section{Christian Reuter*, Volkmar Pipek and Claudia Müller}

University of Siegen, Germany

E-mail: christian.reuter@uni-siegen.de

E-mail: volkmar.pipek@uni-siegen.de

E-mail: claudia.mueller@uni-siegen.de

*Corresponding author

\begin{abstract}
Crisis management requires stakeholders not only to show strategic, organisational preparedness for crisis events (e.g., by systematising and professionalising coping with work), but also to develop skills in dealing with unanticipated events and other stakeholders involved in the same crisis. They should not only rely on established information flows and behaviour patterns, but also be able to deal with situational aspects. This usually involves communication work within and between organisations involved in crisis management. We describe the practice of the crisis communication training of a German Electricity Provider (GEP) and the prototype we developed, implemented and evaluated. The collaborative training tool targets not only the local practice, but also interorganisational training that will allow improving the mutual understanding of communication practices and meeting the information needs of other stakeholders.
\end{abstract}

Keywords: crisis training; crisis communication; crisis management; crisis management systems; computer-supported collaborative learning; CSCL; computer-supported cooperative work; CSCW; emergency management; electricity provider.

Reference to this paper should be made as follows: Reuter, C., Pipek, V. and Müller, C. (2009) 'Avoiding crisis in communication:a computer-supported training approach for emergency management', Int. J. Emergency Management, Vol. 6, Nos. 3/4, pp.356-368.

Biographical notes: Christian Reuter studied Information Systems at the University of Siegen, Germany, and the École Supérieure de Commerce in Dijon, France. He worked at the Institute for Information Systems at the University of Siegen on computer-supported crisis communication management projects. His focus is on IT-supported collaboration in decentralised organisations and technological and fixed mobile convergence. Currently, he is working as an IT Consultant.

Volkmar Pipek studied Computer Science and Economics at the University of Kaiserslautern, Germany, focusing on database systems and artificial intelligence. He worked at the University of Bonn, Germany, and Oulu, Finland, on Human-Computer Interaction (HCI), Computer-Supported Cooperative Work (CSCW) and ubiquitous computing in intra- and 


\begin{abstract}
interorganisational settings. Currently, he is an Assistant Professor for CSCW at the Institute for Information Systems at the University of Siegen, Germany, and leads projects on knowledge work and emergency management.

Claudia Müller is a Research Assistant and a $\mathrm{PhD}$ student in the research group 'CSCW in Organizations' at the University of Siegen, Germany. Her specific interest is in ethnographically informed technology development in complex and dynamic interorganisational information and communication contexts.
\end{abstract}

\title{
1 Introduction
}

Crisis situations often have extensive consequences on the behaviour of the people affected as well as the actors responsible for crisis management. Organisations are trained to operate along established protocols, but there is a large part of the coordination work that goes along more informal lines, and it is based on the individual experience of the crisis managers involved and on the specific characteristics of a crisis. Crisis situations may worsen if inadequate reactions to situations/communications external to organisations occur (Hausschildt et al., 2005). These communication competencies need to be enhanced just like all other competencies involved in crisis management.

This paper presents how Information Technology (IT) can support collaborative training in crisis communication management for an infrastructure-providing company using the example of a German Electricity Provider (GEP). We observed and analysed the existing practice of simulating and practising crisis situations at the GEP, developed a concept and implemented a prototype that aims at integrating stakeholders also from other organisations. From our experiences, we derived some recommendations for computer-supported training in crisis communication.

\section{Training for crisis communication management}

\subsection{Crisis management and communication}

Corporate crises are caused by a constellation of many external and internal factors (Krystek, 2007). Usually, the trigger for the crisis is external, but the reason for the crisis is the (wrong) reaction of the management (Hausschildt et al., 2005). In the case of an electric power company, a breakdown of electricity supply is not only a trigger but can be perceived as a crisis.

Emerging crises can be managed actively using crisis prevention or reactively using coping strategies. Good communication plays an important role in crisis management and consists of a proactive policy (Milis and van de Walle, 2007). If the crisis reaches an advanced stage, the threats will be bigger and the latitude to act will be smaller. Defined organisational structures and existing infrastructures are important elements (Jönck, 2006). Besides this, crisis management training is a crucial element, which has to be prepared like a script for a movie and needs a neutral observer, a logbook and an openness to criticism (Jönck, 2006). Mistakes are often made in the first few hours of a crisis, and it is now possible to simulate them well. For infrastructure companies 
(like GEP), it is also important to have effective information management with state and civil organisations (Murphy and Flournoy, 2002). Accordingly, interorganisational management should also be focused on training.

More and more aspects of operative, maintenance and coping work in technological infrastructures are supported by IT. However, IT is currently used very seldom in crisis management. Milis and van de Walle (2007) examined the use of IT in 250 companies from different sectors. They found that "the level of IT used in crisis management is predominantly related to the presence of a member in the crisis management with an IT background". Neither the size of the company nor the importance of crisis management within this company has any impact on the use of IT.

\subsection{Learning and teaching in crisis management}

Training situations should resemble crisis situations to reflect and improve participants' procedural knowledge. Among the learning theories, constructivist approaches in our eyes relate best to this context (Duffy and Jonassen, 1992). Sociocultural approaches which consider learning as a collective process that is situated in a certain area are also very important (Wenger, 1998).

Strohschneider (2000) compared different approaches to crisis management training. Stress reduction training uses stress immunisation with cognitive restructuring, systematic desensitisation and progressive relaxation. Emergency skills training focuses on the necessary manual abilities to enable people to use their skills even in stressful situations. It should take place in real processes (Keinan et al., 1990). Crew resource management reclaims crisis management competencies in stressful environments. General crisis management group training is especially suitable for low-risk environments, without any danger awareness.

Möhrle and Müller (2005) suggested using the scenario technique. Here, we do not refer to the forecast of a specific situation, but design a spectrum of possible situations. To enhance perceptiveness, crisis triggers are created, that are not very likely but which have a big impact. One challenge is to simulate the individual psychological processes (Sniezek et al., 2002); therefore, we simulate these scenarios. Scenarios are also used in the approach of Benjamins and Rothkrantz (2007), but they are employed in a more technical, nonsocial way.

Computer-supported crisis simulations are one possibility to support scenario-based training. In Computer-Supported Collaborative Learning (CSCL), simulations, micro worlds, hypermedia and gaming systems have proven to be appropriate software types in this context (Pohl, 1999). Simulation games can be realised as computer-supported presence games in which all participants are at the same place at the same time, or as internet-based simulation games. In this paper, the focus will be on the second approach to enable interorganisational communication.

\subsection{Computer-based gaming simulation}

A simulation game provides a learning method that helps one gain experience without any negative impact on reality. It enables the learner to carry out actions without fear or risks that he cannot carry out in reality because analysing them will be too slow, 
too fast, too expensive, too complex or too dangerous (Ruohomäki, 1995). A use is suitable where tasks with many factors and variables have to be trained, especially in emergency and business management (Wagner, 2005).

Simulations can be classified into simulations of natural dependencies and dynamics, and social simulations. For collaborative crisis training, social simulations fit better in focusing on the collaboration of different actors with not always appropriate decisions. Quanjel et al. (1998) found that crisis management training often lacks realistic interaction, objective evaluation and structured feedback. IT can help design a realistic learning context using existing resources, processes, networks and structures, and it can also be used as a communication tool and to record all actions. Recording has benefits and risks. Dron and Bhattacharya (2007) pointed out privacy issues and the perception of control while Kriz (2005) pointed out its usefulness for debriefing.

The process of a simulation game is as follows: In the preparation, the reality is modelled by complexity reduction, and the participants are introduced to the game. The gaming phase includes actions and reactions in a given framework. For the evaluation, it is important to record the solution steps, decisions and results. Afterwards, a comparison of the simulated game experience with the real world has to be made. The aim is to adopt real action patterns, but not malfunctioning patterns, which fit into the game but not into reality (Kriz, 2005).

\section{Empirical study}

Aside from the theory-led consideration above, we aimed to understand the organisation and the practice of the agents by conducting an empirical study. Instead of explicit requirements, we mainly wanted to explore implicit requirements. These are informal information about the process of inter- and intraorganisational crisis management and training, and used artefacts requirements for an integrated crisis-training tool. Those requirements are not usually formulated by the participants. The development of software for supporting collaborative work or learning requires a good (empirical) understanding of the context (Pankoke-Babatz et al., 2001). According to the Standish Chaos Reports, the most prominent reason for a failure within software engineering is a lack of user orientation (Standish Group, 1995).

\subsection{Research field}

The field research for this study took place in one of the biggest private electric power companies in Europe (called GEP here). Experienced members on different levels form the crisis management unit, which operates along an escalation pattern that involves higher hierarchical levels and specialists (e.g., for chemical materials) as a crisis escalates (more people affected, bigger area affected, longer-lasting coping work, etc.). The more a crisis escalates, the more actors need to be involved (e.g., police if traffic lights fail, fire fighters if chemical plants are affected, public administration and the public depending on the spatial and temporal scope of a crisis).

The crisis situations we concentrated on were different scenarios of a power outage, from planned maintenance-related service interruptions to unplanned local power failures (e.g., caused by construction workers) to large regional power outages in relation to extreme weather conditions (floods, heavy ice rains, etc.). A special challenge is that 
other infrastructures depend on the infrastructure 'energy'. Mobile phones are typically available for half an hour, the landline phone is just available for emergency calls, internet and television are not available at all (Sauter, 2006). Different parties (end users, companies, organisations) have different information needs, which may not be served because of missing infrastructures.

\subsection{Empirical methods}

We used qualitative research methods because the criteria and questions were quite open, and our aim was to understand the field. We wanted to be open to new cognitions, which could appear during the research process (Randall et al., 2007). We used a document analysis, observations and group discussions. The aim was to enable a triangulation that would enlarge the validity and reliability of the study (Flick, 2008).

For the document analysis, we analysed ten documents from the electric power company concerning the planning, process and evaluation of the last crisis training and a protocol with observations with an average length of ten pages. Other sources used were ten transcribed interviews with members of the company who were responsible for the requirements of crisis management activities. The aim was to understand crisis training of the company and existing problems and errors. The analysis focused on the preparation, processing and evaluation of crisis training.

We also made participatory observations (Randall et al., 2007) during a two-day workshop about crisis management. The workshop was about 16 hours long, and it had 13 participants from infrastructure engineering and the management of GEP. The aim of the workshop was the creation of an organisational structure for the crisis and the preparation of crisis training. Our observations aimed at getting a rich picture from the organisational structure, the training context and the possibilities for interaction and administration.

The group discussions tried to summarise the statements of all group members. Many opinions about social coherence were expressed in social situations (Mayring, 2002). We had two three-hour-long group discussions with three members of the company and external consultants. The aim was to create explicit requirements for a crisis management system. Besides the explicit methods, we conducted an informal, unstructured data collection in about 25 meetings and workshops during an 18-month research project in the same context.

\subsection{Existing practice of crisis communication training}

When facing a crisis, the company organises a crisis management unit and an adjunct crisis management group for special tasks and assistants. The task of the crisis management group is to make strategic decisions. The adjusted crisis management group consists of members from different departments, who can consult the crisis management group. The task of the assistants is to be an interface for both incoming communication and the actors at the operative level. Representatives of external organisations may be missing, but they are involved if necessary (a list with relevant contacts is maintained). The difficulties of involving actors from other organisations are different crisis vocabularies, different organisational structures and different practices in using communication infrastructures. 
Internal as well as external information sources are used. The internal sources are, for example, screenshots from the local net monitoring system (a highly sophisticated system used to visualise the current state of the power grid, including a sensor network, data lines that operate independently, several monitoring stations including large-screen displays which can show geographic as well as grid-related information as device connection plans), different registers of internal and external contacts, different maps and files with organisational procedures. External sources include information from local fire and police departments and from larger plants that rely on power infrastructures.

The training preparation usually starts with the elaboration on a scenario. It includes different actions at different times. The results are summarised in a PowerPoint presentation. Afterwards, the planned communication routes are designed and put in an Excel sheet. They also create a catalogue with possible questions. External organisations do not participate. During the training, the scenario is played through step by step; new events are submitted via e-mail or fax. Possible questions of external organisations are asked via telephone. The events are recorded into an Excel sheet. Problems with earlier crisis trainings were the plethora of calls, which were not part of the evaluation because they could not be logged. The company discussed the training afterwards, but a systematic evaluation did not follow.

Many activities that are actually done without IT, via PowerPoint or Excel, can be supported with an integrated system. The main requirement is a system which provides an overview of a crisis. This system should create the information from other operative systems. It should not be used like an expert system, and it should be conceived without any precognitions. It should also contain ports to systems of the police and fire brigade. To plan training, it should be possible to create a script. Trainers should also be able to change roles and to define alternatives. Interaction is one main component of the system. It should be possible to communicate with all agents, participating or not. The communication should use the same infrastructures as in a real crisis. An automatic $\log$ can help reclaim the evaluation. Trainers should evaluate reactions and insert comments, which enable them to make a better and more specific evaluation at the end. This information should also be shown with statistical tools.

\section{A concept for computer-supported collaborative training in crisis communication}

We have found both theoretical and practical requirements to collaborative training in crisis communication management. Elements like a proactive policy, a defined organisational structure, infrastructures and crisis simulations are basic requirements. The aim of the training is to achieve the necessary competencies. This process should use constructivist methods and can be realised with social simulations, e.g., simulation games integrated in a crisis management system, using existing data, information, tools and infrastructures. The training can be a combination of different training types, especially crew resource management, to teach nontechnical skills. Another aim is the advance of the use of crisis management systems (emergency skill training) and the reduction of stress (stress reduction training). IT should support group interaction, communication and the modelling, processing, logging and evaluation of the training. 
Roleplaying is one of the main components in creating collaborative awareness. It is totally adaptable and the organisational structures can be mapped dynamically. Agents can participate as themselves or can be simulated. It is also possible to communicate with them, but it is more likely that the trainer or actor will answer. Every agent belongs to one user group and has specific rights. To implement this part of the concept, an agent management has to be created.

To design an authentic training environment, the training system has to be included into a crisis management system. This enables the user to learn how to use real systems, tools, infrastructures and data. It is necessary to enable the trainer to change some context information (like maps or news), to model the training, to make an evaluation, and to record past real situations and use them as one component in the training management of a scenario.

The collaboration with other companies and organisations is important for infrastructure companies. In synchronous and asynchronous interaction with internal and external agents, the compatibility of the vocabulary with the information systems has to be checked. If all communication is integrated in one system, the logging and, therefore, the evaluation are easier. To enable a trainer to play the roles of different actors, patterns for different actors, e.g., fire fighters, press or companies, are required.

The training administration plans the scenario and accomplishes the training. To do this, they plan scenarios in a table and a time bar, which can then afterwards be compared to the real actions. This is currently done on Excel sheets. To support this, an automatic log system and time management can help.

The required functionalities is summarised in Table 1.

Table 1 Required functionalities

\begin{tabular}{|c|c|}
\hline Module & Description \\
\hline User management & Creates, changes, deactivates and deletes agents \\
\hline Role management & $\begin{array}{l}\text { Allocates every agent to a specific role }(e . g ., \text { crisis management, } \\
\text { assistant, team member, external) }\end{array}$ \\
\hline Right management & $\begin{array}{l}\text { Arranges every agent to the hierarchy (e.g., participant, } \\
\text { observer, trainer and administrator) or simulates a user }\end{array}$ \\
\hline Scenario management & $\begin{array}{l}\text { Creates, changes, activates and deletes scenarios for a specific } \\
\text { time and displays them in a table and on a time bar }\end{array}$ \\
\hline Event management & $\begin{array}{l}\text { Allocates events like documents, messages and reactions } \\
\text { to a scenario }\end{array}$ \\
\hline Message management & Creates, prepares, sends, receives and deletes messages \\
\hline Document media management & $\begin{array}{l}\text { Displays and administers documents and media within } \\
\text { categories to create a realistic context for specific target groups }\end{array}$ \\
\hline Verbal communication & Communicates and $\operatorname{logs}$ the attributes of the call in the protocol \\
\hline Geographical energy situation & Gives an overview about current errors \\
\hline Time management & Changes the simulation time in relation to real time \\
\hline Protocol & $\begin{array}{l}\text { Logs all communication within the system, filters it for agents } \\
\text { and media }\end{array}$ \\
\hline
\end{tabular}


Our approach remains more general than the approach of Gomez (2008), which focuses on communication based on Short Message Service (SMS) and uses speech act theory. Our concept focuses on improving the communication competencies of the actors involved as well as improving interorganisational information flows. We also do not aim at an improved 'communication efficiency' through standardisation (e.g., using speech acts), as this makes the most sense in repeating situations while in our considerations, communication becomes particularly valuable with regard to unforeseeable, situational aspects of crisis management. This also distinguishes our approach from Benjamins and Rothkrantz (2007), who also focus on premodelled information in their platform for crisis simulation. Nevertheless, it will be highly interesting to also include these technologies and approaches in a system that aims at practising and building up communication competencies.

\section{Development of a prototype}

To demonstrate the concept, we implemented a prototype. This system supports the whole spectrum of preparing, performing and analysing. With this prototype, it was possible to visualise and evaluate the concept. We conceptualised it as a web architecture to enable users to use it from any place without being required to install software. We used Hypertext Preprocessor (PHP) and My Structured Query Language (MySQL) as programming languages. To use the system intuitively, we chose an iconic representation which eases the finding of functions.

The implementation consisted of a user and role management, a scenario management to model actions, a document and media management to simulate the environment and a message management for the written communication. Apart from that, we implemented an automatic protocol of the whole interaction, integrated a Voice-over Internet Protocol (VoIP) system and a system to display the geographical energy situation.

Figure 1 The user interface (see online version for colours)

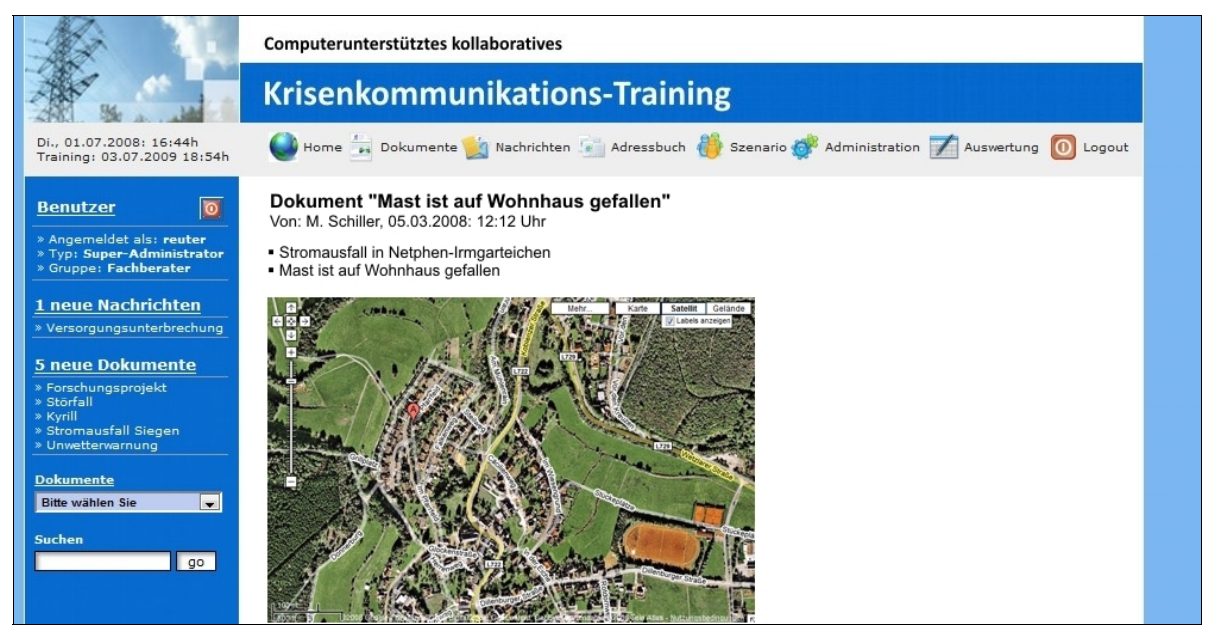


The agent management defines which agents are participating in the training. Messages sent to a simulated agent will be delivered to a trainer. One can always see, highlighted by colours, how many unread messages each user has, helping the trainer judge the participants. The scenario management is for planning and modelling the training. Messages, documents, media and anticipated reactions can be assigned to scenarios. It is possible to prepare a volume of scenarios and trigger them depending on the development of the situation. If one scenario is activated, the actions are carried out automatically.

The message management enables the participants to send electronic messages. Trainers can prepare messages and assign them to a scenario. The verbal communication is realised with an integrated Skype interface. The calls are automatically logged to a protocol.

The document and media management contains documents of all standard formats. The categories represent the ports to other systems, like geographical information, media or data from the information platform of the company. Trainers are able to create documents for a specific target group and simulated agents, and to allocate them to a scenario. Current accidents like planned abandonments, local, regional or big errors can be displayed in an integrated map module, for which GoogleMaps is used.

The communication protocol is the basis for the evaluation of the training. All communications - messages, calls, documents, notes and scenarios - are logged and can be displayed as a time bar. Trainers can review the actions of the participants. Manual protocol entries can fix impressions or anticipated reactions. After the training, the protocol is visible if the communication was done as planned.

Figure 2 Communication protocol as a time bar (see online version for colours)

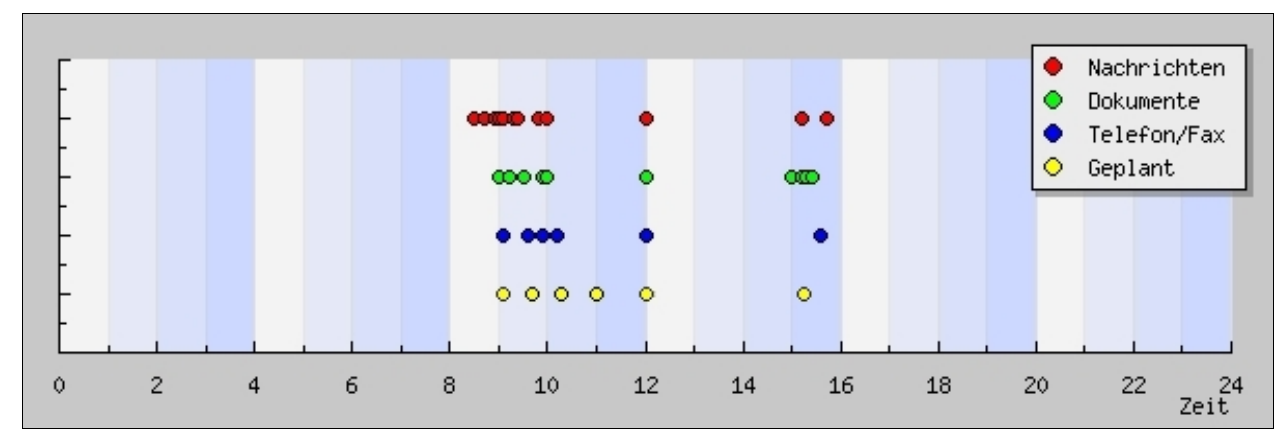

Figure 3 shows the procedure for the training. 
Figure 3 Procedure of computer-supported collaborative training (see online version for colours)

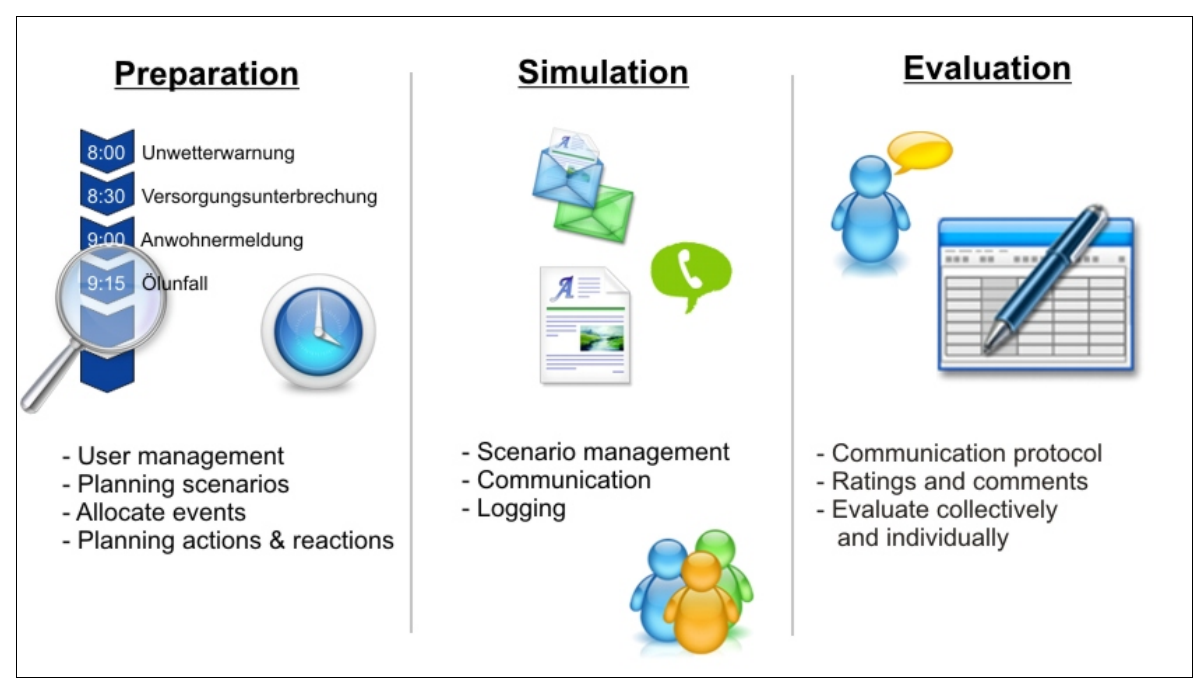

\section{Evaluation}

We did an implicit formative evaluation during the iterative developmental process. We used qualitative methods and integrated the results to the evolutionary process of software development (Cremers et al., 1998). Furthermore, we made a summative evaluation to analyse the system with a scenario-based walkthrough, a technique that directs the user with tasks through the system. To do this, both concept and prototype were presented to three potential users, and we simulated a whole crisis communication management training in about 3 hours. Therefore, we created the users in the system and assigned roles to them. Then we designed scenarios with messages, documents and anticipated reactions. The execution of the training contained activating activities to write messages and simulate the environment with documents, calls and notes. They were all listed automatically in the protocol. Over the course of the entire evaluation, the users were asked to state their impressions while interacting with the system by thinking aloud.

The users were very satisfied and liked preparing, processing and evaluating the whole training within one system, instead of many local documents. The possibility to design the scenarios collaboratively without the explicit exchange of documents improved awareness and usability. The adaptability to different organisational structures and the possibility to model the environment were considered to be very important. The control of the whole scenario at every stage was another important factor. What remained unclear was the ability to know how many documents and messages are allocated to a scenario: "How can I see whether documents belong to a scenario?" The possibility to open a scenario and then to see the documents was easy to understand. The automatic log and further manual protocol entries and comments can create a better basis for an evaluation. Explicit critics are not welcome within this context: "We cannot implement that ... we don't want to critique colleagues." This was a surprising finding because progress is not possible without criticism. Another possibility can be to not criticise the colleagues directly in the system, but to criticise their role names. 


\subsection{Interorganisational aspects}

The original idea and goal of our research was to develop a system that allows different organisations, who need to collaborate in the event of a crisis, to practise collaboratively the communication flows that reflect the particularly situational (i.e., unforeseeable) aspects of a crisis. The training will not only improve the local processes and information flows, but can also contribute to an increased awareness of the information necessities of other organisations that are involved in crisis management. We used GEP as the nucleus of our first experiment, but aimed at a platform that would work independently of the local IT infrastructure. Nevertheless, it will be interesting to closely integrate the system for practising crisis communication into the GEP infrastructure management system itself. To integrate the system to systems that fire fighters, police and other civil organisations use, it needs to provide clear interfaces to these systems. It still requires a significant effort to also include the actors of these organisations, but based on our approach to provide a distributed CSCL platform, the participation costs of each individual organisation may be lowered. The experiences within GEP also show that an interorganisational approach will require sophisticated ways of dealing with event logs and debriefing processes, as critiques resulting from training is considered highly political information.

\section{Conclusion}

In this contribution, we described the development of a collaborative crisis communication training concept and tool for an infrastructure-providing company. In addition to skills and knowledge about coping and recovery work in an actual crisis, crisis managers also need to develop communication skills and train the management of information chains, particularly for crises of a size that requires interorganisational coordination. The training can be supported by simulation and role-playing games. A central element is 'interaction'. IT can support the communication itself and the logging of game events and reactions. Our empirical research tried to answer the questions of how crisis management and communication work in the context of a GEP company and what the specific requirements and the potential of IT support are.

- Crisis management training should contain collaborative communication-oriented elements and scenarios to model the context in advance, but with the opportunity to decide which scenario should arise in the training. An automatic log helps monitor and evaluate the process.

- We also found that systems like ours should be integrated into crisis management systems in order to enable realistic inter- and intraorganisational communication that may even allow training participants to see what others see in the IT systems they use.

- Another particularity of crisis scenarios is that the availability of information and communication systems may not be given in the event of a crisis involving infrastructure providers. Crisis scenario practices should also reflect this type of failure. One important aim for the future is to create technological convergence, especially for critical infrastructures. 
In being better prepared for crisis management, there are many directions one may choose from. While it is important to improve the strategic preparedness of actors in crisis management, e.g., using process modelling and improvement techniques, there will always be situational aspects that the actors need to respond to, and that require in situ coordination and communication, and skills to interact appropriately with others. In further research, we will extend our system with regard to interorganisational requirements towards a stronger integration with crisis management tools. We will also focus on the convergence of critical information infrastructures and explore the consequences of the use of social software in crisis management (Reuter, 2009). The concept as a whole will also be evaluated for other crisis situations.

\section{References}

Benjamins, T. and Rothkrantz, L.J.M. (2007) 'Interactive simulation in crisis management', in B. Van de Walle, P. Burghardt and C. Nieuwenhuis (Eds.) Proceedings of the 4th International Conference on Information Systems for Crisis Response and Management ISCRAM2007, pp.571-580.

Cremers, A.B., Kahler, H., Pfeifer, A., Stiemerling, O. and Wulf, V. (1998) 'PoliTeam - Kokonstruktive und evolutionäre Entwicklung einer Groupware', Informatik-Spektrum, Vol. 21, pp.194-202.

Dron, J. and Bhattacharya, M. (2007) 'Lost in the web 2.0 jungle', in J.M. Spector, D.G. Sampson, T.O. Kinshuk, S.A. Cerri, M. Ueno and A. Kashihara (Eds.) 7th IEEE International Conference on Advanced Learning Technologies (ICALT), IEEE Computer Society, Niigata, Japan, Vol. 7, pp.895-896.

Duffy, T.M. and Jonassen, D.H. (1992) Constructivism and the Technology of Instruction: A Conversation, Hillsdale: Lawrence Erlbaum Associates.

Flick, U. (2008) Triangulation: Eine Einführung, Wiesbaden: VS Verlag für Sozialwissenschaften.

Gomez, E.A. (2008) 'Crisis response communication management: increasing message clarity with training over time', in F. Fiedrich and B. Van de Walle (Eds.) Proceedings of the 5th International Conference on Information Systems for Crisis Response and Management ISCRAM2008, pp.368-375.

Hausschildt, J., Grape, C. and Schindler, M. (2005) 'Typologien von Unternehmenskrisen im Wandel', Schriftenreihe aus den Instituten für Betriebswirtschaftslehre der Universität Kiel $(C A U)$, p.588.

Jönck, U. (2006) 'Krisenkommunikation', in T. Hutzschenreuter and T. Griess-Nega (Eds.) Krisenmanagement. Grundlagen - Strategien - Instrumente, Wiesbaden: Gabler, pp.447-462.

Keinan, G., Friedland, N. and Sarig-Naor, V. (1990) 'Training for task performance under stress: the effectiveness of phased training methods', Journal of Applied Social Psychology, Vol. 20, pp.1514-1529.

Kriz, W.C. (2005) 'Gaming simulation - state of the art', in U. Blötz (Ed.) Planspiele in der beruflichen Bildung, Bielefeld: Bertelsmann, Vol. 4, pp.247-256.

Krystek, U. (2007) Handbuch Krisen- und Restrukturierungsmanagement, Generelle Konzepte, Spezialprobleme, Praxisberichte, Kohlhammer.

Mayring, P. (2002) Einführung in die Qualitative Sozialforschung, Weinheim/Basel: Beltz Verlag.

Milis, K. and van de Walle, B. (2007) 'IT for corporate crisis management: findings from a survey in 6 different industries on management attention, intention and actual use', HICSS 2007, p.24.

Möhrle, M.G. and Müller, S. (2005) 'Frühzeitige Krisenwahrnehmung durch Szenarien und anknüpfende Methoden', in C. Burmann, J. Freiling and M. Hülsmann (Eds.) Management von Ad-hoc-Krisen: Grundlagen- Strategien- Erfolgsfaktoren, Gabler Verlag. 
Murphy, W.S., Jr. and Flournoy, M.A. (2002) 'Weapon and communication systems: simulating crisis communications', Winter Simulation Conference, pp.954-959.

Pankoke-Babatz, U., Prinz, W., Wulf, V. and Rohde, M. (2001) 'Spezifika des CSCW-Designs', in G. Schwabe, N. Streitz and R. Unland (Eds.) CSCW-Kompendium, Lehr- und Handbuch zum computerunterstützten kooperativen Arbeiten, Berlin: Springer, pp.373-393.

Pohl, C. (1999) Methodik und Realisation von Systemen zur effizienten Wissensvermittlung durch Hypermedia, Frankfurt am Main: Peter Lang Verlag.

Quanjel, M.M.H., Willems, A.J. and Talen, A.N. (1998) 'CRISISLAB: Evaluation and improvement of crisis management through simulation/gaming', Simulation Gaming, Vol. 29, pp.450-455.

Randall, D.W., Harper, R.H.R. and Rouncefield, M. (2007) Fieldwork for Design: Theory and Practice, London: Springer.

Reuter, C. (2009) 'Social Software als kritische Informations-Infrastruktur', in S. Kain, D. Struve and H. Wandke (Eds.) Workshop-Proceedings der Tagung Mensch \& Computer 2009, Berlin: Logos-Verlag, pp.140-144.

Ruohomäki, V. (1995) 'Viewpoints on learning and education with simulation games', in J.O. Riis (Ed.) Simulation Games and Learning in Production Management, London.

Sauter, M. (2006) Grundkurs Mobile Kommunikationssysteme: Von UMTS und HSDPA, GSM und GPRS zu Wireless LAN und Bluetooth Piconetzen, Vieweg+Teubner.

Sniezek, J.A., Wilkins, D.C., Wadlington, P.L. and Baumann, M.R. (2002) 'Training for crisis decision making: psychological issues and computer-based solutions', Journal of Management Information Systems Archive, Vol. 18, pp.147-168.

Standish Group (1995) 'The Standish Group report', http://www.educause.edu/ir/library/pdf/ NCP08083B.pdf.

Strohschneider, S. (2000) Handeln in Krisensituationen - Anforderungen, Fehler, Trainingsansätze, Bamberg: Institut für Theoretische Psychologie, Otto-Friedrich-Universität Bamberg.

Wagner, I. (2005) Einsatz und Evaluation eines nutzergerechten Autorensystems für webbasierte Planspiele, Kassel: Kassel University Press.

Wenger, E. (1998) Communities of Practice: Learning, Meaning, and Identity, Cambridge: Cambridge University Press. 